\title{
Damage to Type II Collagen in Aging and Osteoarthritis Starts at the Articular Surface, Originates Around Chondrocytes, and Extends into the Cartilage with Progressive Degeneration
}

Anthony P. Hollander, ${ }^{*}$ Isabelle Pidoux, ${ }^{\star}$ Agnes Reiner, ${ }^{*}$ Cecil Rorabeck, ${ }^{\star}$ Robert Bourne, ${ }^{\star}$ and A. Robin Poole

* Joint Diseases Laboratory, Shriners Hospital for Crippled Children, Division of Surgical Research, Department of Surgery, McGill University, Montreal, Canada, H3G 1A6; and ${ }^{\ddagger}$ Division of Orthopaedic Surgery, University Hospital, University of Western Ontario, London, Ontario, N6A 5A5 Canada

\section{Abstract}

Enhanced denaturation of type II collagen fibrils in femoral condylar cartilage in osteoarthritis (OA) has recently been quantitated immunochemically (Hollander, A. P., T. F. Heathfield, C. Webber, Y. Iwata, R. Bourne, C. Rorabeck, and A. R. Poole. 1994. J. Clin. Invest. 93:1722-1732). Using the same antibody that only reacts with denatured type II collagen, we investigated with immunoperoxidase histochemistry (results were graded for analysis) the sites of the denaturation (loss of triple helix) of this molecule in human aging (at autopsy, $n=11$ ) and progressively degenerate (by Mankin grade [MG]) OA (at arthroplasty, $n=51$ ) knee condylar cartilages.

Up to 41 yr, most aging cartilages (3 of 4) (MG 0-4) showed very little denaturation. In most older cartilages, (4 of 7) (MG 2-4), staining was observed in the superficial and mid zones. This pattern of collagen II denaturation was also seen in all OA specimens with increased staining extending to the deep zone with increasing MG. Collagen II staining correlated directly both with MG and collagen II denaturation measured by immunoassay. Cartilage fibrillation occurred in OA cartilages with increased penetration of the staining for collagen II denaturation into the mid and deep zones and where denaturation was more pronounced by immunoassay.

Thus in both aging and OA the first damage to type II collagen occurs in the superficial and upper mid zone (low MG) extending to the lower mid and deep zones with increasing degeneration (increasing MG). Initial damage is always seen around chondrocytes implicating them in the denaturation of type II collagen. (J. Clin. Invest. 1995. 96:2859-2869.) Key words: cartilage $\cdot$ collagen - osteoarthritis • denaturation $\bullet$ immunohistochemistry

Address correspondence to A. Robin Poole, Joint Diseases Laboratory, Shriners Hospital, 1529 Cedar Avenue, Montreal, Quebec, H3G 1A6, Canada. Phone: 514-849-6208; FAX: 514-842-5581. Anthony Hollander's present address is Department of Human Metabolism and Clinical Biochemistry, University of Sheffield Medical School, Sheffield, S10 2RX United Kingdom.

Received for publication 15 June 1994 and accepted in revised form 16 August 1995.

J. Clin. Invest.

(C) The American Society for Clinical Investigation, Inc.

$0021-9738 / 95 / 12 / 2859 / 11 \quad \$ 2.00$

Volume 96, December 1995, 2859-2869

\section{Introduction}

The degeneration of articular cartilage is a key feature of osteoarthritis $(\mathrm{OA})^{1}$. It is characterized by a loss of the tensile strength $(1-3)$ of this tissue. The incidence of OA increases progressively with age (4). This is in association with a progressive reduction in tensile properties during aging (5). Whereas the large aggregating proteoglycan (now called aggrecan) is responsible for the compressive stiffness of cartilage $(6,7)$, it is the collagen fibrillar network that determines the tensile properties of this tissue $(1,2)$. These collagen fibrils are primarily composed of type II collagen ( 90-95\%) but also contain type IX collagen, covalently bound to type II, and type XI collagen which together comprise $2-4 \%$ of the total collagen (8).

These biomechanical changes are indicative of damage to the collagen fibrils in aging and OA. To investigate these changes at the molecular level first polyclonal (9) and then monoclonal (10) antibodies were developed that only react with epitopes on the collagen $\alpha_{1}$ (II) chains when the triple helix has been denatured, such as occurs following cleavage by interstitial collagenase (MMP1) which results in unwinding of the triple helix.

Using a monoclonal antibody in an immunoassay, quantitative evidence was obtained to indicate that there is indeed increased denaturation of type II collagen in osteoarthritic cartilage (10). In the study presented here we describe the use of this antibody to determine where, in both aging and osteoarthritis, this damage to type II collagen occurs. We show that it starts at the articular surface and spreads into the mid and deep zones with increasing degeneration, denaturation being first observed around chondrocytes implicating these cells as primary mediators of collagen degradation in aging and OA.

\section{Methods}

Tissue. Full depth human articular femoral condylar cartilages (0.5-1.0 $\mathrm{cm}^{2}$ surface area) were removed within $15 \mathrm{~h}$ of death with a sharp scalpel from the anterior loaded regions of the adult knee joint from a total of 11 individuals, of various ages and sexes, with no observable arthritic joint abnormalities nor recent (2-3 mo) chemotherapy (see Table I). A total of 51 patients underwent total knee arthroplasty for osteoarthritis diagnosed using the criteria of the American College of Rheumatology. Site matched femoral condylar cartilages that remained were immediately removed to the laboratory. By definition, osteophytic cartilages were excluded from this study. Single samples from 30 patients (see Table I) and 12 multiple samples from three other patients

1. Abbreviation used in this paper: OA, osteoarthritis. 

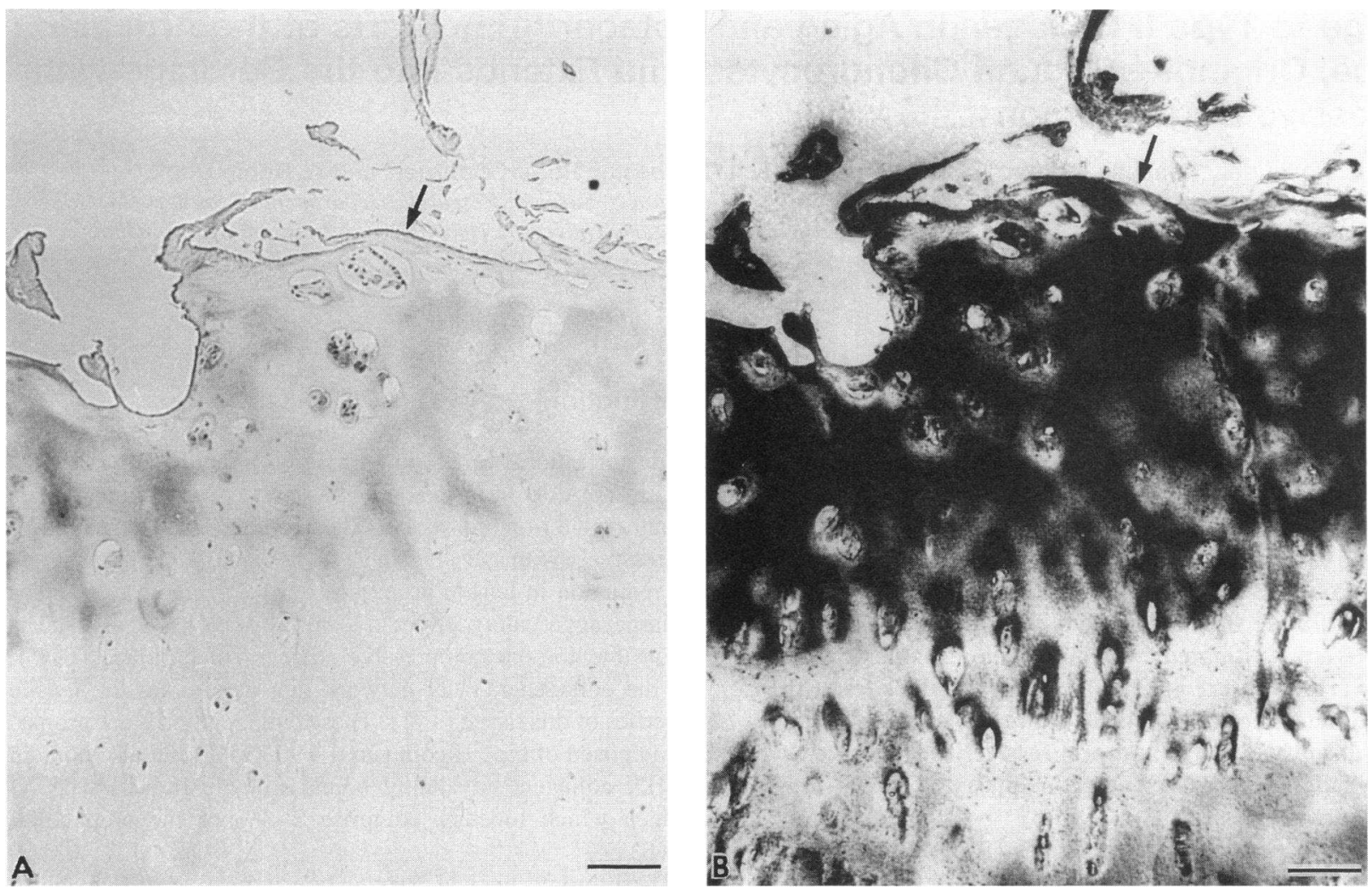

Figure 1. Specificity of monoclonal antibody COL2-3/4m. Osteoarthritic cartilage stained with antibody preabsorbed with $(B)$ peptides CB11A (epitope absent) or $(A)$ CB11B (epitope present). Staining is essentially abolished by peptide CB11B. Arrow indicates articular surface. Scale $=100 \mu \mathrm{m}$.

(see Table II) were used in detailed analyses of interrelationships of age, sex, type II collagen denaturation (by immunohistochemistry and immunoassay), and Mankin grade. The cartilages from 21 other patients were used only to investigate collagen denaturation by immunohistochemistry and Mankin grade.

Monoclonal antibody to denatured type II collagen and immunoassays of total type II collagen content and collagen II denaturation. Monoclonal antibody COL2-3/4m, a mouse $\operatorname{IgG}_{1}(\kappa)$ that reacts with an epitope in denatured, but not native, type II collagen was described previously (10). It recognizes a 13 residue amino acid sequence which is located in the CB11 region of type II collagen from all species so far studied.

Peptide CB11B (C-G-K-V-G-P-S-G-A-P(OH)-G-E-D-G-R-P(OH)G-P-P(OH)-G-P-Q-Y) which contains the epitope, was synthesized at a $0.25-\mathrm{mmol}$ scale, using standard Fmoc chemistry, on a solid phase peptide synthesizer (model 431 A; Applied Biosystems, Inc., Foster City, CA (10). A second peptide $\alpha 1$ (II)-CB11A (C-G-P-Q-G-A-P(OH)-GP-A-G-E-E-G-K-R-G-A-R-G-E-P(OH)-Y) that lacks the epitopes was also synthesised and used as a control peptide in immunohistochemical studies.

Immunoassays of total type II collagen content and percentage denaturation were conducted as described previously (10).

Immunohistochemistry. Tissue blocks were mounted in OCT embedding media (Miles Laboratories, Naperville, IL), rapid frozen and 6$\mu \mathrm{m}$ thick sections were cut at $-20^{\circ} \mathrm{C}$ using a Tissue-Tek II cryostat. Sections were picked up on glass microscope slides precoated with aminoalkylsilane as described by Henderson (11), to ensure complete adherence of the tissue sections to the slides. This is very important in these studies since our monoclonal antibody will detect denatured type II collagen at the cut surfaces of the section. Incomplete adherence is revealed by very weak patches of staining throughout the specimen (see
Fig. $2 \mathrm{~A}$ ). Thus complete adherence is essential to ensure uniformity of immunoreactivity. Specific staining of type II collagen denatured by cutting with a scalpel was also observed at the cut junction with calcified cartilage and wherever the specimen had been cut to remove it from the cartilage. The articular surface was left untouched so that the collagen denaturation we recorded had occurred naturally and was not artifactually induced.

Sections were either immediately frozen and stored at $-20^{\circ} \mathrm{C}$ or used immediately. Similar results were obtained in either case. The sections were fixed for $5 \mathrm{~min}$ in $4 \%$ formaldehyde, freshly prepared from paraformaldehyde in PBS (12), and washed in several changes of PBS for $15 \mathrm{~min}$. These and subsequent manipulations were at room temperature. Unreactive aldehyde groups were blocked with $1 \% \mathrm{wt} / \mathrm{vol}$ normal pig serum in PBS for $15 \mathrm{~min}$. Endogenous peroxidase activity was blocked by incubation of the sections with freshly prepared $0.5 \%$ ( vol/vol) $\mathrm{H}_{2} \mathrm{O}_{2}$ in absolute methanol for $10 \mathrm{~min}$ followed by washing in PBS for $15 \mathrm{~min}$. To enhance permeability of the extracellular matrix, sections were treated with chondroitinase ABC (ICN/Flow Laboratories, Mississauga, Canada ) at $0.0125 \mathrm{U} / 50 \mu \mathrm{l}$ per section in $0.1 \mathrm{M}$ Tris-

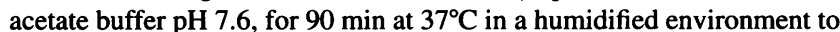
remove chondroitin sulfate (12). After washing in PBS for $15 \mathrm{~min}$ the sections were incubated with $0.2 \mathrm{M}$ EDTA in $50 \mathrm{mM}$ Tris, $\mathrm{pH} 7.6$ for $1 \mathrm{~h}$ at room temperature to ensure removal of any calcific deposits in the specimens. They were then washed for 15 min with PBS containing $0.1 \% \mathrm{wt} /$ vol BSA.

Sections were incubated for $30 \mathrm{~min}$ at room temperature in a humidified chamber with $50 \mu \mathrm{l} / \mathrm{section}$ of monoclonal antibody COL $2-3 / 4 \mathrm{~m}$ ascitic fluid (diluted 1/100) or control, nonimmune ascitic fluid, each diluted in PBS containing 1\% wt/vol BSA. More specific controls were prepared by incubating monoclonal antibody COL2 $-3 / 4 \mathrm{~m}$ before use for $1 \mathrm{~h}$ at $37^{\circ} \mathrm{C}$ with $100 \mu \mathrm{g} / \mathrm{ml}$ of its epitope contained in peptide 

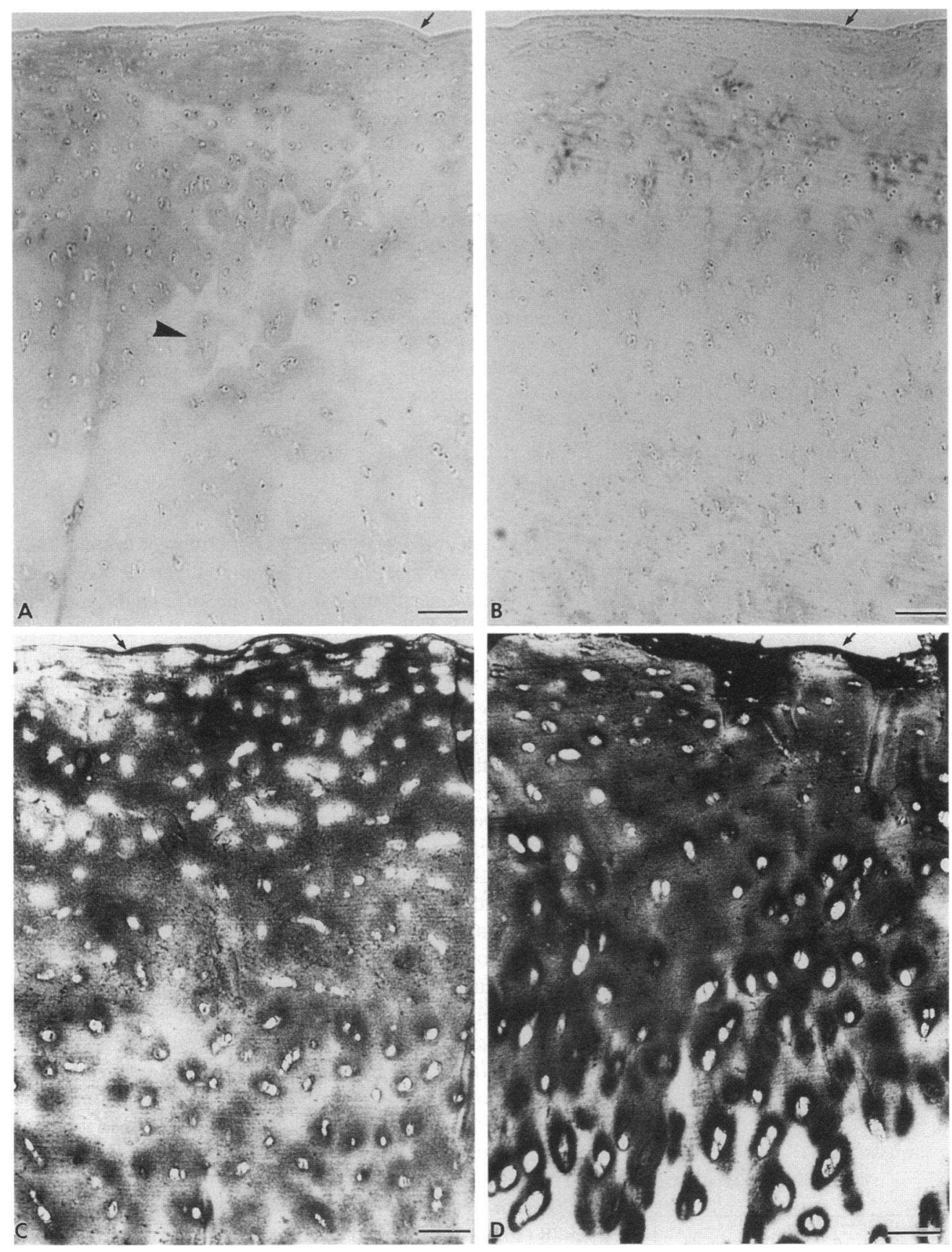

Figure 2. Nonarthritic cartilages stained with monoclonal antibody COL2-3/4m . (A) specimen, 41 yr, Mankin grade 1 , collagen grade $0.5 ;(B)$ specimen, $28 \mathrm{yr}$, Mankin grade 1 , collagen grade 0.5 ; $(C)$ specimen, $57 \mathrm{yr}$, Mankin grade 4 , collagen grade $3 ;(D)$ specimen, $81 \mathrm{yr}$, Mankin grade 2, collagen grade 2 . Weak staining caused by poor attachment to slide is indicated (arrowhead) in A. Arrow indicates articular surface. Scale $=100 \mu \mathrm{m}$.

CB11B, or an unrelated peptide CB11A of the same length. Sections were washed three times for $10 \mathrm{~min}$ each with PBS containing $0.1 \%$ wt/vol BSA. A biotin-streptavidin detection system (Amersham Corp., Arlington Heights, IL) was used according to the manufacturers recommendations. This consisted of pig $\mathrm{F}\left(\mathrm{ab}^{\prime}\right)_{2}$ anti-mouse Ig labeled with biotin and peroxidase-conjugated streptavidin as the indicator. Biotinylated pig anti-mouse $\left.\mathrm{F}\left(\mathrm{ab}^{\prime}\right)\right)_{2}$ was diluted in PBS with $1 \%$ wt/vol BSA at $50 \mu \mathrm{l} / \mathrm{section}$ for $30 \mathrm{~min}$. After washing with azide-free PBS, the sections were treated with $50 \mu \mathrm{l} /$ section of the diluted peroxidase-conjugated streptavidin in azide-free PBS with $1 \% \mathrm{wt} / \mathrm{vol}$ BSA for $20 \mathrm{~min}$ at room temperature. The sections were washed twice for $10 \mathrm{~min}$ in azide-free PBS, postfixed by incubating with $2 \% \mathrm{vol} / \mathrm{vol}$ glutaraldehyde in azide-free PBS and then stored in $50 \mathrm{mM}$ Tris, $\mathrm{pH} 7.6$, for $24 \mathrm{~h}$. The peroxidase reaction was developed and the product was enhanced with copper $-\mathrm{H}_{2} \mathrm{O}_{2} /$ silver intensification as previously described (13). Permanent mounts were prepared.

Grading of immunohistochemical staining for type II denaturation (collagen grading). Three to four full depth sections of each specimen were examined. No staining was recorded as 0 . Traces of staining mainly in pericellular sites was graded as 0.5 (see Fig. 2, $A$ and $2 B$ ). Definitive staining restricted to the superficial layer and (where present) upper mid zone was recorded as 1 (see Fig. $3 A$ ). Diffuse staining of the superficial zone and of the mid zone with pericellular staining of the upper deep zone was graded as $2^{+}$(see Fig. $3 B$ ). Similar staining to 2 that extended throughout the depth of the deep zone was designated 3 (see Fig. $3 C$ ). Staining of most of the extracellular matrix throughout the depth of the cartilage, with loss of staining in the superficial zone associated with fibrillation, was recorded as "4" (see Fig. 5A). In 
Table I. Patients Studied and Relationships between Mankin Grade and Overall Staining for Type II Collagen Denaturation

\begin{tabular}{|c|c|c|c|c|c|}
\hline Patient & Age & Sex & & $\begin{array}{l}\text { Mankin grade } \\
\quad(0-13)\end{array}$ & $\begin{array}{c}\text { Collagen } \\
\text { staining }(0-4)\end{array}$ \\
\hline Nonarthritic & & & Cause of death & & \\
\hline N1 & 35 & $\mathbf{F}$ & pulmonary fibrosis & 0 & 0.5 \\
\hline N2 & 28 & $\mathrm{~F}$ & $\begin{array}{l}\text { ethylene glycol } \\
\text { poisoning }\end{array}$ & 1 & 0.5 \\
\hline N3 & 41 & $\mathbf{M}$ & cardiac arrest & 1 & 0.5 \\
\hline N4 & 58 & $\mathbf{M}$ & pulmonary embolism & 1 & 1 \\
\hline N5 & 66 & $\mathbf{M}$ & pancreatic cancer & 1 & 0.5 \\
\hline N6 & 67 & $\mathbf{M}$ & leukemia & 1 & 0.5 \\
\hline N7 & 81 & $\mathbf{F}$ & sepsis & 2 & 2 \\
\hline N8 & 69 & $\mathbf{M}$ & myocardial infarction & 3 & 0.5 \\
\hline N9 & 34 & $\mathbf{M}$ & pulmonary fibrosis & 3 & 1 \\
\hline N10 & 57 & $\mathbf{F}$ & pulmonary fibrosis & 4 & 3 \\
\hline N11 & 69 & $\mathbf{F}$ & myocardial infarction & 4 & 2 \\
\hline Mean \pm SD & & & & $1.91 \pm 1.38$ & $1.05 \pm 0.91$ \\
\hline Osteoarthritic & & & Removed at ar & rthroplasty & \\
\hline OAl & 71 & $\mathbf{M}$ & & 1 & 1 \\
\hline $\mathrm{OA} 2$ & 69 & $\mathbf{M}$ & & 3 & 1 \\
\hline OA3 & 65 & $\mathbf{F}$ & & 3 & 2 \\
\hline OA4 & 76 & $\mathbf{M}$ & & 3 & 3 \\
\hline OA5 & 52 & $\mathbf{M}$ & & 4 & 3 \\
\hline OA6 & 71 & $\mathbf{M}$ & & 4 & 2 \\
\hline OA7 & 62 & $\mathrm{~F}$ & & 5 & 3 \\
\hline OA8 & 52 & $\mathbf{M}$ & & 5 & 1 \\
\hline OA9 & 82 & $\mathbf{M}$ & & 5 & 2 \\
\hline OA 10 & 76 & $\mathrm{~F}$ & & 5 & 3 \\
\hline OA11 & 62 & $\mathrm{~F}$ & & 6 & 3 \\
\hline OA12 & 74 & $\mathbf{M}$ & & 6 & 3 \\
\hline OA13 & 68 & $\mathbf{M}$ & & 6 & 3 \\
\hline OA14 & 86 & $\mathrm{~F}$ & & 7 & 2 \\
\hline OA15 & 71 & $\mathbf{M}$ & & 7 & 2 \\
\hline OA16 & 68 & $\mathbf{M}$ & & 7 & 3 \\
\hline OA17 & 73 & $\mathrm{~F}$ & & 7 & 4 \\
\hline OA 18 & 74 & $\mathrm{~F}$ & & 8 & 2 \\
\hline OA19 & 82 & $F$ & & 8 & 3 \\
\hline OA20 & 71 & $\mathbf{F}$ & & 8 & 3 \\
\hline OA21 & 69 & $\mathbf{M}$ & & 9 & 3 \\
\hline OA22 & 74 & $\mathbf{F}$ & & 9 & 2 \\
\hline OA23 & 70 & $\mathbf{M}$ & & 9 & 4 \\
\hline OA24 & 71 & $\mathbf{M}$ & & 9 & 4 \\
\hline OA25 & 86 & $F$ & & 10 & 4 \\
\hline OA26 & 55 & $\mathrm{~F}$ & & 10 & 4 \\
\hline OA27 & 63 & $\mathbf{M}$ & & 10 & 4 \\
\hline OA28 & 76 & $F$ & & 10 & 4 \\
\hline OA29 & 73 & $\mathbf{M}$ & & 11 & 4 \\
\hline OA30 & 63 & $\mathbf{F}$ & & 11 & 4 \\
\hline Mean $\pm S D$ & & & & $7.00 \pm 2.62^{*}$ & $2.93 \pm 0.92^{*}$ \\
\hline
\end{tabular}

* Significantly different by Mann-Whitney $U$ analyses, $P=0.0001$. A single specimen was removed from each individual.

all these studies the immunohistochemical staining was performed in triplicate to ensure data reproducibility.

Mankin grading. Frozen sections prepared as described above were stained with Safranin $O$ and Fast green and graded for degenerative changes as described by Mankin et al. (14). This did not include analysis of the calcified cartilage that was absent from the specimens. Hence the maximum grade was 13 . These grades are indicated in Tables I and II and in the figure legends. They were correlated with other analyses. The Mankin grade refers only to that of the specimen examined by immunohistochemistry. Adjacent specimens were analyzed for collagen denaturation by immunoassay.

Interobserver variation: recording of Mankin and collagen II denaturation gradings by immunohistochemistry. Two independent observers (A. R. Poole and I. Pidoux ) independently examined the same random- ized blinded specimens (normal, $n=11$; OA, $n=32$ ) independently. Data were then compared by Spearman rank correlation to determine variation in data recording. The results for Mankin grading were $r$ $=0.933, P=0.0001$. Those for collagen grading were $r=0.877, P$ $=0.0001$. These results reflect good reproducibility of analyses between observers. The results of one blinded observer (I. Pidoux) are reported in this study.

Staining for proteoglycan with toluidine blue. This was performed using $0.5 \%$ toluidine blue in $5 \%$ ethanol in water. After 10 min excess stain was removed by washing in water. Permanent mounts were prepared.

Photomicrographs. The results that are shown are for full depth or almost full depth specimens of articular cartilage unless otherwise stated.

Statistical analyses. Specimen rank correlations were used for analyses of possible relationships between the different parameters recorded in this study. Mann-Whitney $U$ tests were used to compare normal and OA groups.

\section{Results}

The results of detailed analyses of the reactivity of this antibody with normal and osteoarthritic femoral condylar cartilages are described below. Sections stained with Safranin $O$ and Fast green were graded for degenerative changes as described by Mankin (14). Other sections were analyzed for collagen II denaturation with antibody COL $2-3 / 4 \mathrm{~m}$.

Specificity of antibody COL2-3/4m reactivity in immunohistochemistry and reproducibility of staining. Sequential sections of an osteoarthritic cartilage were treated with the monoclonal antibody after incubation with either peptide CB11B (containing epitope) or CB11A (epitope absent). Staining was only observed in Fig. $1 B$ after incubation with peptide CB11A. Prior reactivity of the antibody with the epitope in peptide CB11B prevented binding to the section as shown in Fig. $1 \mathrm{~A}$. A similar lack of staining to that obtained with peptide CB11B was observed when other OA specimens were examined or when nonimmune ascitic fluid or purified nonimmune mouse $\operatorname{IgG}_{1}$ were used (data not shown).

Staining with antibody COL2-3/4m of nonarthritic femoral condylar cartilages from patients with no macroscopic evidence of joint disease. To establish the amount and location of the reactivity of the antibody COL2-3/4m with normal articular cartilage, sections were examined from various individuals of age $20-40 \mathrm{yr}(n=3) 41-50 \mathrm{yr}(n=1) 51-60 \mathrm{yr}(n=2)$, 61-70 yr $(n=4), 71-90 \mathrm{yr}(n=1)$. Of these, three persons aged 28 (Fig. $2 \mathrm{~B}$ ), 35, and 41 yr (Fig. $2 A$ ) showed very little collagen staining $(0.5)$ (Mankin grades 0 or 1 ) (Table I). Some nonspecific stain that was detected was very faint and was due to antibody binding to the cut surfaces of the frozen sections due to incomplete adherence of the section to the glass slide. The arrowhead in Fig. $2 A$ for example shows some of this very weak artifactual staining in territorial and interterritorial sites. The pattern of this very weak staining varied in sequential sections: it was more pronounced when diethylsilane was not used to enhance adherence to the glass slide. The weak but stronger staining observed around chondrocytes was not due to sectioning artifacts (Fig. $2 B$ ). This was always noted close to the articular surface as shown. Only one patient (N9) of four patients in this age range up to 41 yr exhibited clearly defined collagen staining (1, Mankin grade 3$)$ : this person suffered from pulmonary fibrosis (Table I).

With increasing age, Mankin grade remained at 1 or in- 

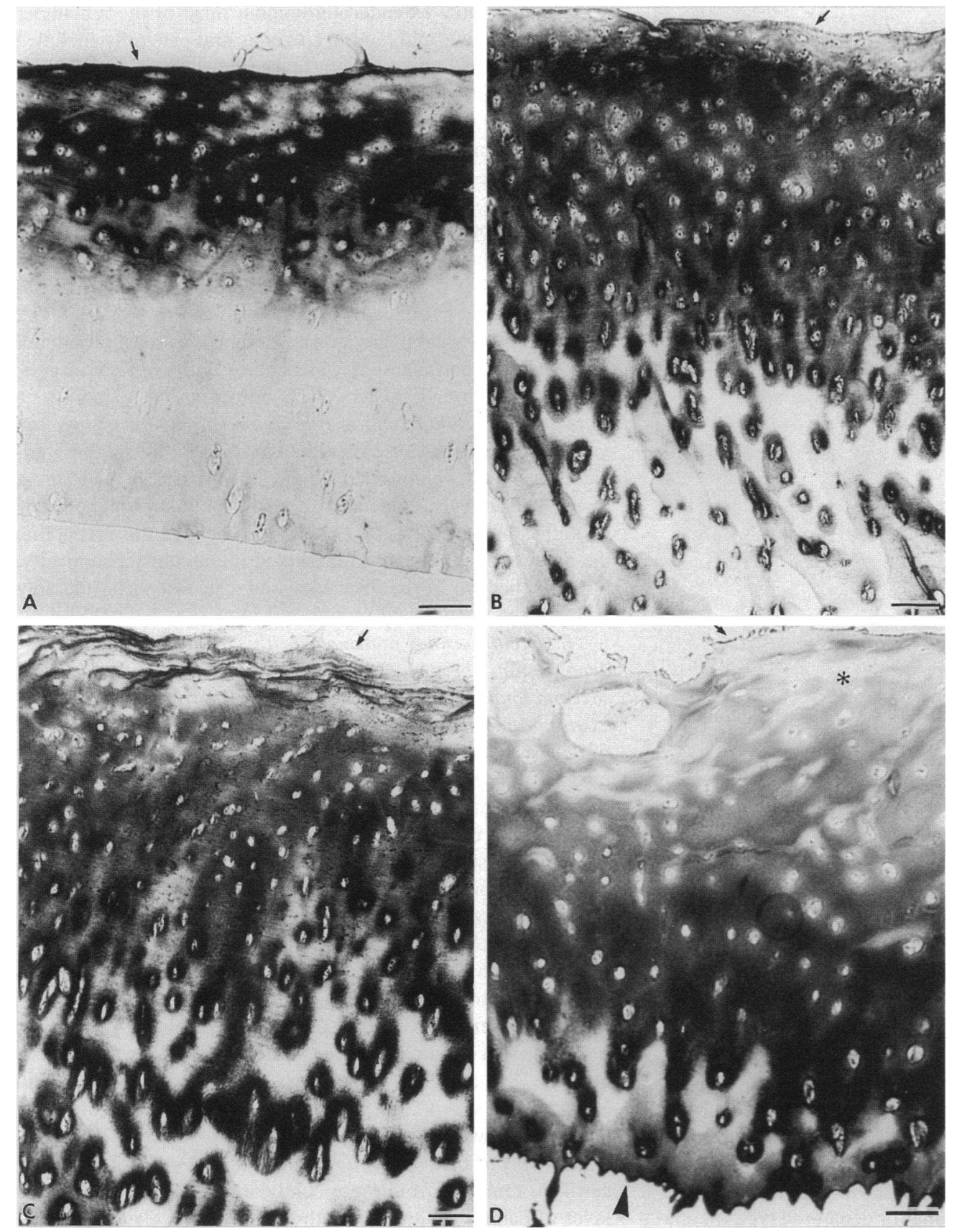

Figure 3. Osteoarthritic cartilages (Mankin grades 4 and 5) stained with monoclonal antibody COL2$3 / 4 \mathrm{~m}(A)$ specimen, Mankin grade 4 , collagen grade $1 ;(B)$ specimen, Mankin grade 5 , collagen grade $2 ;(C)$ specimen, Mankin grade 5 , collagen grade $3 ;(D)$ specimen Mankin grade 5, collagen grade 4 . Superficial cartilage matrix showing very reduced staining is indicated (asterisk). The articular surface of the cartilage is indicated by arrow. Scale $=100 \mu \mathrm{m}$.

creased in one person to grade 2 , in two individuals to 3 , and in two to grade 4. Cartilages with a Mankin grade of 2 (Fig. 2 $D$ ) and 4 (Fig. $2 C$ ) exhibited abundant staining for denatured collagen but never greater than collagen grade 3 . This extended from the articular surface into the mid and deep zones where staining was most pronounced around chondrocytes. The articular surface was essentially intact. Spearman rank analysis revealed that there were no correlations between collagen staining grade and Mankin grade, nor of either of these variables with age or sex (data not shown). Relationships to collagen II denaturation were not determined since this assay was not performed in this group.

Staining with antibody COL2-3/4m of osteoarthritic cartilage removed at arthroplasty. A total of 64 specimens from 51 different patients were examined. The group in which detailed correlative analysis were performed for collagen denaturation, Mankin grade, age, and sex are shown in Table I. Another group of 21 patients was used for immunolocalization of collagen II denaturation and Mankin grade only. These results are shown in Figs. 3, 4, 5, 7, and 8. The Mankin grades (range 1-11) either overlapped or were higher than those seen in the nonarthritic group (range 0-4) (Table I). They were significantly higher, however, in the OA group (Table I). Staining for denatured type II collagen was also significantly increased in OA than in the nonarthritic group (Table I).

Examination of Mankin grades 4 and 5 revealed staining patterns not unlike those observed in the nonarthritic cartilages of older nonarthritic persons shown in Fig. 2, $C$ and $D$. In the 


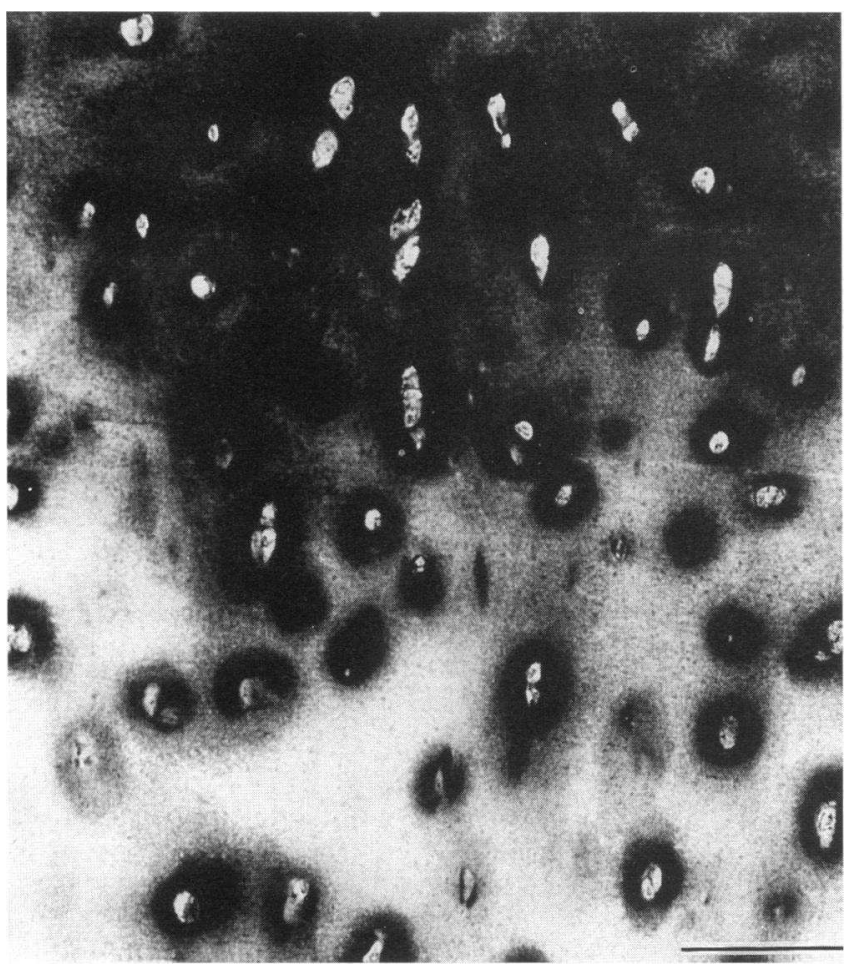

Figure 4. Osteoarthritic cartilage Mankin grade 4 stained with monoclonal antibody COL2-3/4m to show collagen denaturation adjacent to chondrocytes at junction of more damaged collagen in mid zone with that showing primarily damage around chondrocytes in deep zone. Scale $=100 \mu \mathrm{m}$.

specimen shown in Fig. $3 A$ (Mankin grade 4) strong staining was observed but this was restricted to the superficial and upper mid zones, with the deeper zone being unstained even in pericellular sites. Specimens of grade 5 (Fig. 3, $B$ and $C$ ) showed patterns of staining resembling that observed in the 81-yr-old nonarthritic cartilage (Fig. 2 D). But in Fig. 3, $A-D$ there was generally less staining in the most superficial cartilage where evidence for early fibrillation was observed. Fibrillation was more developed in some specimens (Fig. $3 D$ ) and this was accompanied by a further reduction in staining under the articular surface compared to that seen deeper in the cartilage.

A striking feature of this staining for denatured type II collagen was that in the deeper layers where staining was present, it was mainly confined to pericellular sites (Fig. 3, $A-D$ ). When transition zones, located between stained and unstained cartilage, were examined in normal and OA cartilages (Mankin grade 4, Fig. 4) it was clear that the staining was indeed confined to pericellular and territorial sites indicating that the damage to the collagen was first observed around the chondrocytes and not remote from them. Also in some specimens where collagen staining involved much of the depth of the specimen, more selective staining of the cut edge in a deep zone, in which staining was less pronounced, was observed (see Fig. $3 \mathrm{D}$, arrowhead).

With progressive degeneration of the articular cartilage (Fig. 5, $A$ and $C$ ) more superficial and deep fibrillation was often observed. This was again accompanied by a consistent loss of staining at the remaining articular surface (Fig. 5, A-
C). Staining now extended throughout most of the remainder of the superficial, mid, and deep zones and generally included the pericellular, territorial, and interterritorial sites of the deep zone. This pattern was usually only observed at Mankin grades of 7 or more with exceptions as shown in Fig. 5 A (Mankin grade 4). Sometimes staining was reduced or absent in the mid and upper deep zones as shown in Fig. $5 B$. In other cases, as shown in Fig. 5, $A, C$, and $D$, reduced staining was observed around chondrocytes. This may represent severely damaged matrix in which collagen epitopes have been lost from damaged molecules, as is probably the case at the articular surface. In very degenerate cartilage of high grade, such as specimen OA 30 in Fig. $5 D$ (Mankin grade 11), staining was seen throughout the remaining cartilage but was again usually weaker in pericellular sites around clusters of chondrocytes.

Local variation in collagen II denaturation (by immunohistochemistry and immunoassay) and Mankin grade. Within individual condyles we observed significant variation in the Mankin grade and collagen II denaturation by immunohistochemistry as well as by immunoassay (Table II). These results reveal that the process of degeneration measured immunohistochemically, histochemically, or by immunoassay can vary considerably within a relatively small region of a joint.

Interrelationships in osteoarthritic cartilages of collagen II denaturation (by immunohistochemistry), Mankin grade, age, sex, and total collagen II content and denaturation (by immunoassay). Analyses were performed of the specimens described in Tables I and II. Spearman rank analyses revealed that neither type II collagen denaturation (immunohistochemistry) nor denaturation (immunoassay) were correlated with age or sex in osteoarthritic cartilages (Table III). Yet Mankin grade showed a marginally significant direct correlation with age but not with sex in OA cartilages. In contrast, collagen denaturation measured by immunohistochemistry and by immunoassay were significantly directly correlated with Mankin grade in OA (Table III and Fig. 6, $B$ and $C$ ). Moreover, collagen II denaturation detected by immunoassay and collagen II denaturation by immunohistochemistry were also directly correlated (Table III, Fig. $6 \mathrm{~A}$ ). Total type II collagen content showed no correlations in these studies (data not shown). There was no distinction between the sex of the patient and the correlation observed (data not shown).

OA specimens containing pannus tissue. Very occasionally cartilage removed from these condylar sites was covered by a layer of pannus tissue. In fact this represents a classifying feature of the Mankin grading system. In such specimens (Fig. 7) cartilage matrix staining was usually absent to moderate (Fig. $7 \mathrm{~B}$ ) or moderate (Fig. $7 \mathrm{~A}$ ). However, in contrast to other OA cartilages these specimens were characterized by essentially diffuse staining throughout the cartilage matrix quite unlike the patterns described above where more pericellular and territorial staining was also observed. Thus this pattern of damage is different and is associated with the presence of pannus on the cartilage.

Relationship of collagen II denaturation recorded immunohistochemically to proteoglycan (aggrecan) content measured by histochemistry. Serial sections were stained with antibody COL2-3/4m for collagen II denaturation or with toluidine blue which detects primarily the large proteoglycan aggrecan. In specimens such as N1, (Mankin grade 0 ) in which little or no staining was observed with antibody COL2-3/4m (Fig. $8 \mathrm{~A}$ ), 

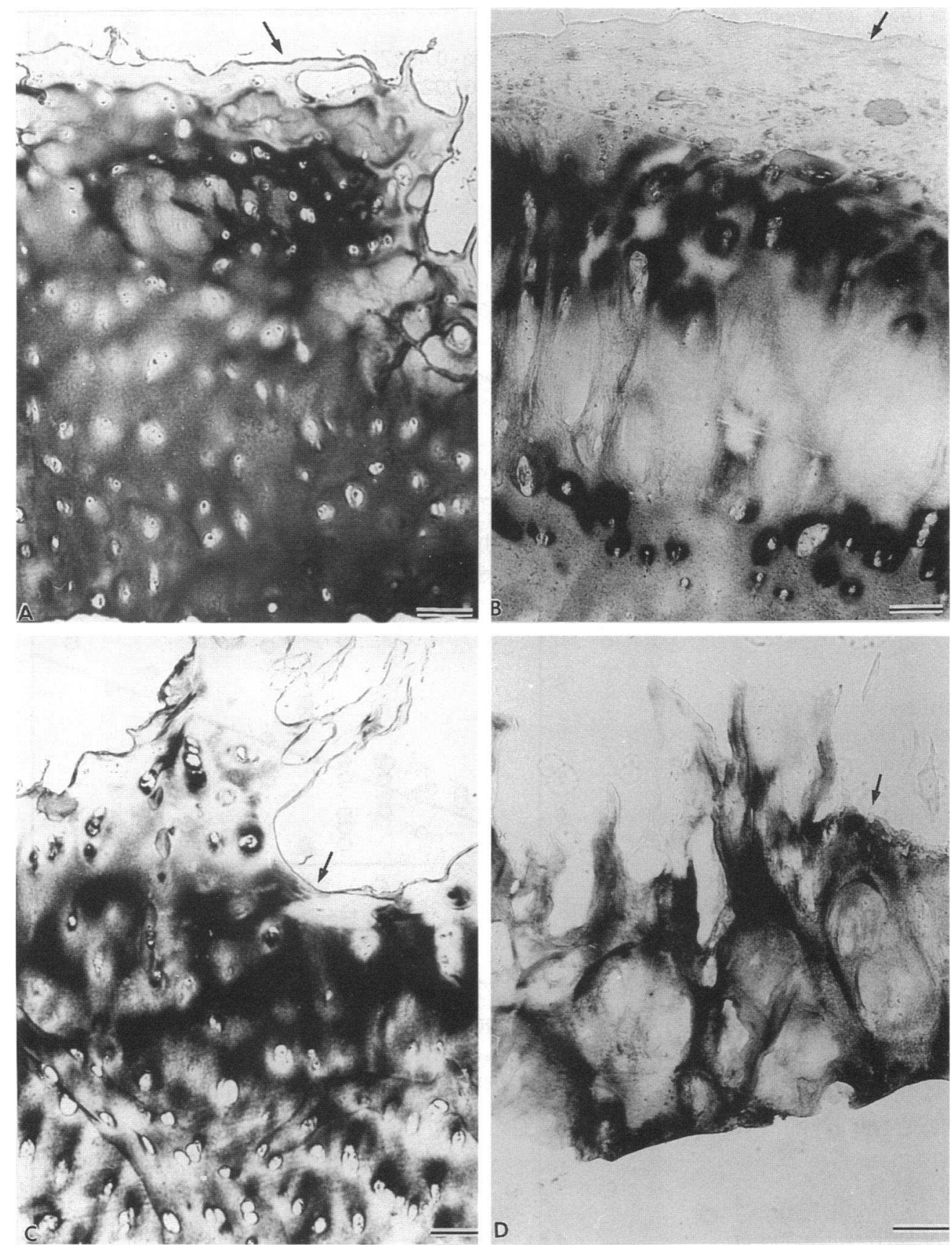

Figure 5. Osteoarthritic cartilages Mankin grades 4-11 stained with monoclonal antibody COL2-3/4m to show more advanced degeneration and fibrillation. $(A)$ specimen, Mankin grade 4, collagen grade 4; $(B)$ specimen, Mankin grade 8 , collagen grade $3 ;(C)$ specimen, Mankin grade 7, collagen grade 3; $(D)$ specimen, OA Mankin grade 11 , collagen grade 4 . The remaining articular surface is indicated by arrows. Scale $=100 \mu \mathrm{m}$.

toluidine blue stained the matrix strongly almost up to the articular surface (Fig. $8 \mathrm{~B}$ ). In contrast, damage to collagen II at and near the articular surface, in OA specimens (Mankin grade 4) (Fig. $8 C$ ) was accompanied by a loss of staining with toluidine blue (Fig. $8 \mathrm{D}$ ). This dye instead stained mainly the matrix around chondrocytes particularly in the interzone between that staining for collagen denaturation and that showing no staining with the monoclonal antibody (Fig. $8 \mathrm{C}$ ).

\section{Discussion}

Earlier analyses of the biomechanical properties of articular cartilage drew attention to the preferential loss of tensile proper- ties of more superficial articular cartilage in aging $(5,15)$ and in early OA (7) around sites of overt fibrillation and so-called ulcerated cartilage $(1,2)$. The present study provides a direct molecular demonstration that it is in the more superficial sites that type II collagen damage (denaturation) is first observed both in aging as well as in OA. With increased degeneration of the cartilage, reflected by an increased Mankin grading, fibrillation is first observed at the articular surface where this denaturation is first detected and then extends down into the cartilage. Together these observations suggest that in the absence of a recognizable molecular defect or joint trauma, articular cartilage degeneration in OA involves a process of molecular denaturation of type II collagen characterized by progressive denaturation of the triple helix of type II collagen and the associated 
Table II. Local Variability in Mankin Grade and Staining for Collagen Denaturation within a Femoral Condyle

\begin{tabular}{|c|c|c|c|c|c|}
\hline \multicolumn{2}{|c|}{ Specimen } & \multirow{2}{*}{$\begin{array}{l}\text { Grading, Mankin, and } \\
\text { collagen II denaturation }\end{array}$} & \multicolumn{3}{|c|}{ Sites } \\
\hline Age & Sex & & 1 & 2 & 3 \\
\hline \multirow[t]{3}{*}{58} & $\mathbf{M}$ & Mankin & 4 & 2 & 3 \\
\hline & & Collagen, IH & 3 & 2 & 3 \\
\hline & & Collagen, IA & 3.7 & 3.0 & 4.1 \\
\hline \multirow[t]{3}{*}{74} & $\mathbf{F}$ & Mankin & 5 & 2 & 4 \\
\hline & & Collagen, IH & 3 & 2 & 2 \\
\hline & & Collagen, IA & 4.6 & 2.7 & 4.5 \\
\hline 51 & $\mathbf{M}$ & Mankin & 4 & 3 & - \\
\hline \multirow[t]{2}{*}{ Left medial } & & Collagen, IH & 3 & 2 & - \\
\hline & & Collagen, IA & 2.9 & 3.2 & - \\
\hline \multirow[t]{2}{*}{51} & $\mathbf{M}$ & Mankin & 12 & 3 & - \\
\hline & & Collagen, IH & 4 & 3 & - \\
\hline Left lateral & & Collagen, IA & 9.7 & 3.2 & - \\
\hline
\end{tabular}

Specimens were located in sites $0.5-2.0 \mathrm{~cm}$ distant from each other within a condyle. $51 \mathrm{M}$ represents specimens from one patient. Collagen II grading by immunohistochemistry $(I H)$ is shown with percent denaturation by immunoassay $(I A)$.

loss of tensile properties of this cartilage that are ordinarily and primarily determined by this molecule. The present observations are complemented by our recent data for collagen II denaturation in human articular cartilage obtained by immunoassay with the same COL2-3/4m antibody used here. These studies also show that not only is there increased denaturation in OA cartilage but that in the majority of OA cartilages and in some nonarthritic (normal) cartilages, denaturation is usually more pronounced in the superficial and mid zones than in the deep zone (10). Our current analyses of OA cartilages clearly reveal that the grading of collagen II denaturation by immunohistochemistry directly correlates with our quantitative analyses by immunoassay as well as with increased degeneration recorded by the Mankin grading system. In fact we show direct links between these three sets of analyses and thereby demonstrate the value of this immunohistochemical approach to study collagen II denaturation.

Table III. Spearman Rank Analyses of Interrelationships in Patients with Osteoarthritis

\begin{tabular}{lccc}
\hline \multicolumn{1}{c}{ Analysis } & $r$ & $P$ & $n$ \\
\hline Age vs & & & \\
$\quad$ Mankin grade & 0.353 & 0.0349 & 28 \\
$\quad$ Collagen II denaturation grade & 0.131 & 0.4460 & 28 \\
$\quad$ Percent denatured type II collagen & 0.128 & 0.4550 & 28 \\
Mankin grade vs & & & \\
$\quad$ Collagen II denaturation grade & 0.681 & $<0.0001^{*}$ & 42 \\
$\quad \begin{array}{l}\text { Percent denatured type II collagen } \\
\text { Collagen grade vs }\end{array}$ & 0.484 & $0.0013^{*}$ & 42 \\
$\quad$ Percent denatured type II collage & 0.463 & $0.0022^{*}$ & 42 \\
\hline
\end{tabular}

* Significant correlations. There were no significant correlations for normal specimens for age, Mankin grade, or collagen grade.
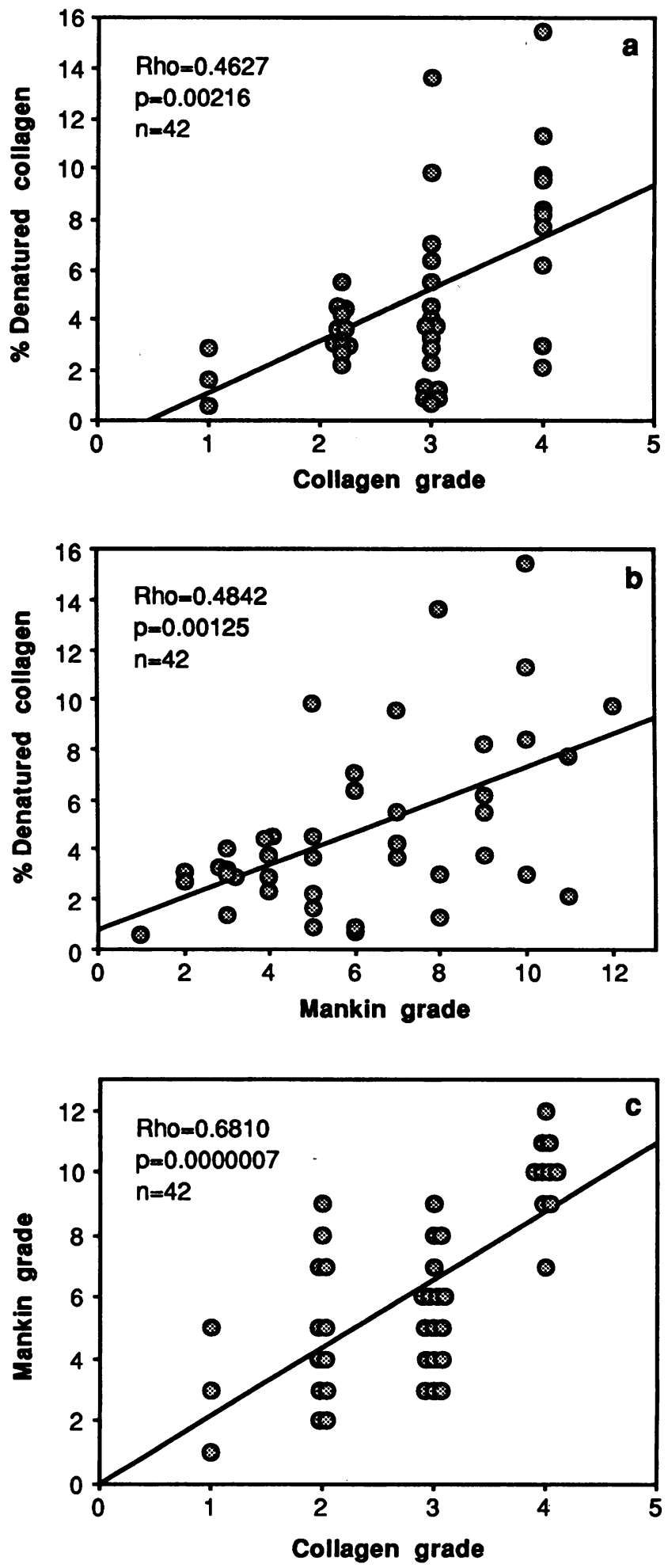

Figure 6. Spearman rank correlations between collagen grade, Mankin grade, and collagen denaturation measured by immunoassay.

The similar staining patterns for collagen II denaturation seen in nonarthritic cartilages that stained for collagen II denaturation and $\mathrm{OA}$ cartilages point to a possible common pathway of progressive damage to type II collagen in the extracellular 

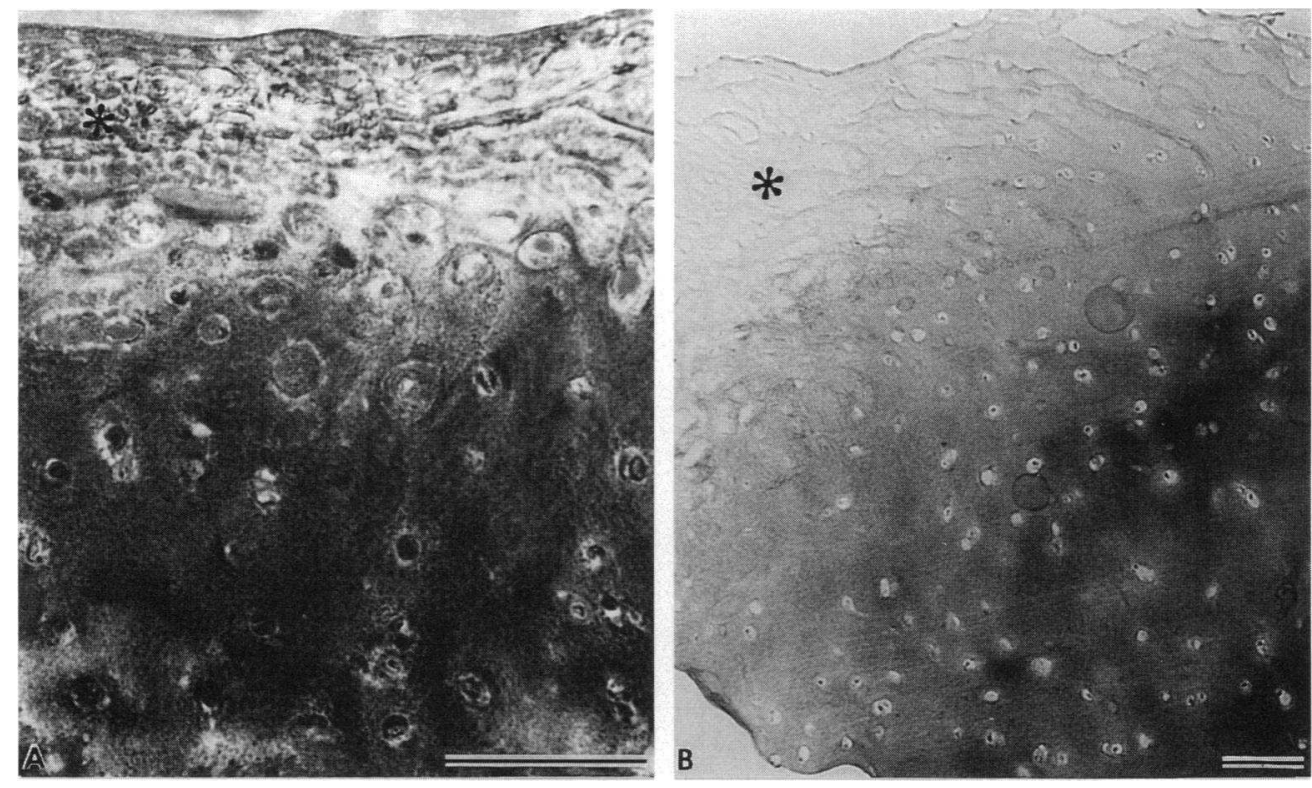

Figure 7. Osteoarthritic cartilages associated with overlying pannus tissue (asterisk) stained with monoclonal antibody COL2-3/ 4m; $(A)$ specimen, OA Mankin grade 6 , collagen grade $4 ;(B)$ specimen, OA Mankin grade 4 , collagen grade 4 ; Scale $=100 \mu \mathrm{m}$.

matrix that originates in the more superficial layers and extends progressively, in an age independent manner, into the mid and deep zones with increasing degeneration of the cartilage recorded by Mankin grade or collagen denaturation by immunoassay. That this involves degradation mediated by the chondrocytes is indicated by the strong pericellular staining that is first observed at the type II collagen "denaturation front." Such pericellular and territorial damage to type II collagen is also induced when healthy human articular cartilage is cultured with IL-1 $(9,16)$. Thus the chondrocyte would appear to have the capacity to denature type II collagen both in vitro and in vivo.

Although we have no definitive data from these human studies presented here of the mechanisms responsible for this damage we have recently shown that collagen damage of this kind induced in vitro by IL-1 can be arrested by a nontoxic hydroxamate inhibitor of metalloproteinases (16). Moreover since collagenases are the only known proteinases in cartilage capable of cleaving the triple helix that is required to produce the denaturation (unwinding) of the triple helix observed and detected here (8), it is likely that this denaturation results from cleavage by a proteinase with the properties of collagenase. At present we know of the existence of three collagenases: interstitial collagenase 1 (MMP-1), neutrophil collagenase (collagenase 2 or MMP-8), and the recently described equivalent to the rodent collagenase (collagenase 3 or MMP-13) (17). Any of these may be involved in the cleavage. Studies of the expression of mRNA for metalloproteinases have revealed that in normal as well as in OA cartilages expression of stromelysin (18) and the 92-kD gelatinase (19), and to a lesser extent collagenase (18), is most pronounced in these more superficial sites where collagen II damage is observed. Moreover staining with antibodies to stromelysin has also revealed that cells in these sites contain more detectable protein in OA (20). Metalloproteinase activity capable of degrading the proteoglycan aggrecan is also generally elevated in OA cartilage (21). Thus sites of early type II collagen damage and loss of aggrecan coincide with the sites where synthesis of these metalloproteinases is likely most pronounced. Since these proteinases are not stored in chondrocytes, these observations suggest therefore that secretion and activity may also be enhanced in these sites.

The reason for the differences in staining observed in two specimens in which pannus is present is not known. This pattern of diffuse staining throughout the cartilage more resembles that which is often observed in articular cartilages from patients with rheumatoid arthritis where pannus tissue is frequently present (Hollander, A., I. Pidoux, E. Bogoch, W. Fisher, and A. R. Poole, unpublished results). It is conceivable that cytokines such as IL1 or TNF $\alpha$ (acting upon chondrocytes) or proteinases such as collagenase originating from this pannus tissue, (acting directly on matrix ) may be more responsible for the damage observed in these specimens. This remains to be established.

These studies also reveal that the characteristic loss of metachromatic staining for aggrecan observed in more superficial sites in articular cartilage in early degeneration of cartilage in $\mathrm{OA}$ is associated with damage to type II collagen. This raises questions of the relationship of collagen II damage to the loss of the proteoglycan. The simple demonstration that loss of the proteoglycan aggrecan (detected by the absence of staining with toluidine blue) selectively occurs in the more superficial sites where collagen fibrils are damaged, points to the importance of maintaining the integrity of this fibrillar collagen network in retaining aggrecan. Analyses have revealed that aggrecan molecules bind to hyaluronic acid to form macromolecular aggregates both in isolation (22) and in situ (23). These aggregates are thought to be retained in the extracellular matrix by some sort of binding to the collagen fibrillar network since regular points of attachment of hyaluronic acid to collagen fibrils have been described (23). Thus it appears that when damage occurs to these fibrils this may be accompanied by a loss of aggrecan. Independent quantitative studies confirming this superficial loss of aggrecan will be reported elsewhere (Poole, A. R, A. Reiner, M. Ionescu, E. Brooks, and E. Bogoch, manuscript in preparation ). Moreover, we have also found that the small proteoglycan decorin, normally concentrated in these more superficial sites in nonarthritic cartilages (24), is lost from these superficial sites when there is damage to type II collagen in OA (Poole, A. R, 

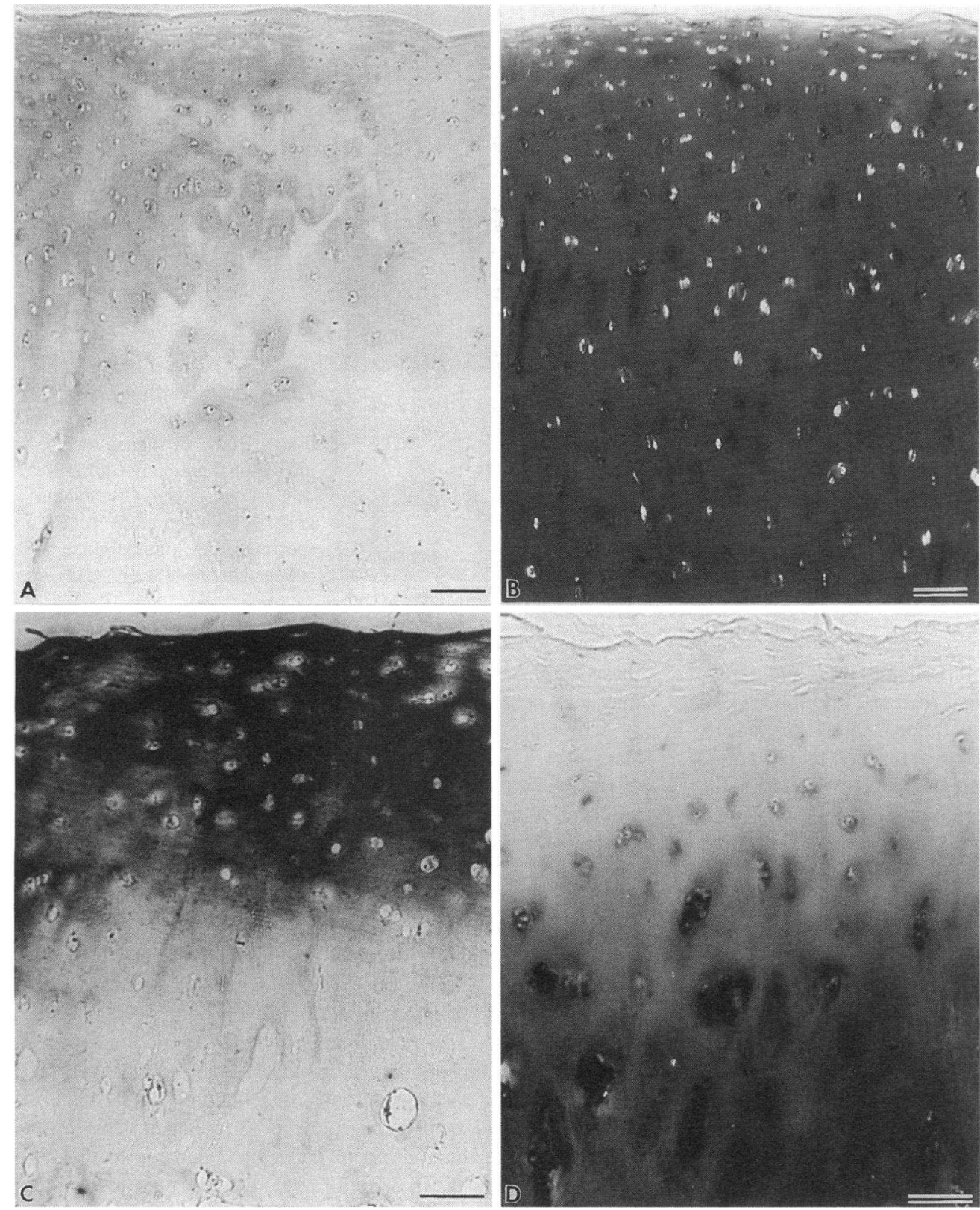

Figure 8. Normal and osteoarthritic cartilage stained with monoclonal antibody COL $2-3 / 4 \mathrm{~m}$ $(A$ and $C)$ and toluidine blue $(B$ and $D)$. $(A)$ specimen, N1 Mankin grade 0 , collagen grade 0.5 ;

$(C$ and $D$ ) specimen, OA Mankin grade 4 and collagen grade 2 represent two sets of sections.

Whereas little or no collagen II staining is seen in the normal cartilage in $(A)$ staining for proteoglycan aggrecan extends up to the articular surface. In contrast in osteoarthritic cartilage the increased damage to type II collagen seen at and close to articular surface in $(C)$ is accompanied by reduced staining for proteoglycan $(D)$. Scale $=100 \mu \mathrm{m}$.

L. C. Rosenberg, A. Reiner, M. Ionescu, E. Bogoch, and P. J. Roughley, unpublished observation). Decorin not only influences assembly of collagen fibrils (25) but ordinarily also binds to them (26). Thus damage to collagen can have profound effects on the retention of other matrix molecules.

It is worth commenting on the apparent lack of staining for collagen denaturation such as we sometimes observed in more degenerate specimens in more superficial sites and around some chondrocytes, particularly in the midzone. The lack of staining in sites where fibrillation has developed is preceded by much increased staining in superficial nonfibrillated OA cartilage. Thus it would appear that in these pericellular sites where staining is lacking but otherwise present in this region, the type II collagen is less denatured than in other sites. This certainly may be the case in pericellular and territorial sites around mid and deep zone chondrocytes. We have evidence from studies of collagen synthesis that it is in these sites in which the C-propeptide of type II collagen is usually observed in OA (Nelson, F., A. Reiner, E. Brooks, and A. R. Poole, manuscript in preparation). Since this C-propeptide content has been shown to directly correlate with collagen II synthesis this reduced staining may be reflective of a net increase in synthesis of type II collagen in a matrix in which significant damage may have previously occurred. This indication of a repair process in response to collagen damage remains to be explored further.

Whether this progressive damage to extracellular type II collagen results from the activity of cytokines such as IL-1 and TNF $\alpha$ changing chondrocyte-mediated matrix turnover or/and from biomechanical alterations in chondrocyte loading remains to be established. But it is likely that once these changes are initiated, leading to a loss of tensile properties (due to collagen damage) and compressive stiffness of the matrix (through ag- 
grecan loss), altered loading of chondrocytes will result. This altered loading can also change in vitro turnover of both aggrecan and type II collagen (27-29). Thus matrix degeneration may progressively involve adjacent chondrocytes in more healthy matrix by a combination of biomechanical alterations and related changes in cytokine and proteinase production both by chondrocytes and possibly also by synovial cells. This may explain the progressive involvement of healthy cartilage in the process of cartilage degeneration in aging and OA as part of a process of creeping substitution, as shown here, resulting in the formation of a matrix containing denatured collagen II that replaces a once healthy functional matrix. Moreover, our observations suggest that these mechanisms may be similar in both aging and $\mathrm{OA}$ with the latter representing more advanced degenerative changes resulting in clinically recognizable degeneration.

\section{Acknowledgments}

The manuscript was processed by Audrey Wheeler.

This study was funded by the Shriners of North America, the Medical Research Council of Canada and Pfizer Central Research (to A. R. Poole). Dr. Hollander was a recipient of a post-doctoral fellowship from the Arthritis Society of Canada.

\section{References}

1. Kempson, G. E., H. Muir, C. Pollard, and M. Tuke. 1973. The tensile properties of the cartilage of human femoral condyles-related to the content of collagen and glycosaminoglycans. Biochim. Biophys. Acta. 297:465-472.

2. Akizuki, S., V. C. Mow, F. Muller, J. C. Pita, and D. S. Howell. 1986. Tensile properties of human knee joint cartilage. I. Influence of ionic conditions, weight bearing, and fibrillation on the tensile modulus. J. Orthop. Res. 4:379392.

3. Kempson, G. E. 1979. Mechanical properties of articular cartilage. In Adult Articular Cartilage. M.A.R. Freeman, editor. Pitman Books Ltd., Tunbridge Wells, Kent, United Kingdom, 333-414.

4. Felson, D. T. 1988. Epidemiology of hip and knee osteoarthritis. Epidemol. Rev. 10:1-28.

5. Kempson, G. E. 1991. Age-related changes in the tensile properties of human articular cartilage. A comparative study between the femoral head of the hip joint and the talus of the ankle joint. Biochim. Biophys. Acta. 1075:223-230.

6. Kempson, G. E. H. Muir, M. A. R. Freeman, and S.A.V. Swanson. 1970. Correlations between the compressive stiffness and chemical constituents of human articular cartilage. Biochim. Biophys. Acta. 215:70-77.

7. Mow, V. C., A. Ratcliffe A., and A. R. Poole. 1992. Cartilage and diarthrodial joints as paradigms for hierarchical materials and structures. Biomaterials. 13:67-97.

8. Poole, A. R. 1993. Cartilage in health and disease. In Arthritis and Allied Conditions. A Textbook of Rheumatology. Vol. 1 12th Ed. D.J. McCarty and W.J. Koopman, editors. Lea and Febiger, Philadelphia. 279-333.

9. Dodge, G. R., and A. R. Poole. 1989. Immunohistochemical detection and immunochemical analysis of type II collagen degradation in human normal, rheumatoid and osteoarthritic articular cartilages and in explants of bovine articular cartilages cultured with interleukin-1. J. Clin. Invest. 83:647-661.

10. Hollander, A. P., T. F. Heathfield, C. Webber, Y. Iwata, R. Bourne, C.
Rorabeck, and A. R. Poole. 1994. Increased damage to type II collagen in osteoarthritic articular cartilage detected by a new immunoassay. J. Clin. Invest. 93:1722-1732.

11. Henderson, C. 1989. Aminoalkylsilane: an inexpensive simple preparation for slide adhesion. J. Histotechno. 12:123-124.

12. Poole, A. R., I. Pidoux, A. Reiner, L.-H. Tang, J. Choi, and L. Rosenberg. 1980. Localization of proteoglycan monomer and link protein in the matrix of bovine articular cartilage. J. Histochem. Cytochem. 28:621-635.

13. Gallyas, F., and I. Merchanthaler. 1988. Copper- $\mathrm{H}_{2} \mathrm{O}_{2}$ oxidation strikingly improves silver intensification of the nickel-diamiobenzidine (Ni-DAB) end product of the peroxidase reaction. J. Histochem. Cytochem. 36:807-810.

14. Mankin, H. J., H. Dorfman, L. Lippiello, and A. Zarins. 1971. Biochemica and metabolic abnormalities in articular cartilage from osteoarthritic human hips. II. Correlation of morphology with biochemical and metabolic data. J. Bone Jt. Surg. Am. Vol. 53A:523-537.

15. Kempson, G. E. 1982. Relationship between the tensile properties of articular cartilage from the human knee and age. Ann. Rheum. 41:508-511.

16. Mort, J. S., G. R. Dodge, P. J. Roughley, J. Liu, S. J. Finch, G. Dipasquale, and A. R. Poole. 1993. Direct evidence for active metalloproteinases mediating matrix degradation in interleukin-1 stimulated human articular cartilage. Matrix. 13:95-102.

17. Freije, J. M. P., I. Diez-Itza, M. Balbin, L.M . Sánchez, R. Blasco, J. Toliva, and C. López-Otin. 1994. Molecular cloning and expression of collagenase-3, a novel human matrix metalloproteinase produced by breast carcinomas. J. Biol. Chem. 269:16766-16773.

18. Nguyen, Q., J. S. Mort, and P. J. Roughley. 1992. Preferential mRNA expression of prostromelysin relative to procollagenase and in situ localization in human articular cartilage. J. Clin. Invest. 89:1189-1197.

19. Mohtai, M., R. L. Smith, D. J. Schurman, Y. Tsuji, F. M. Torti, N. I. Hutchinson W. G. Stetler-Stevenson, and G. I. Goldberg. 1993. Expression of 92$\mathrm{kD}$ type IV collagenase/gelatinase (gelatinase B) in osteoarthritic cartilage and its induction in normal human articular cartilage by interleukin 1. J. Clin. Invest. 92:179-185.

20. Okada, Y., M. Shinmei, O. Tanako, K. Naka, A. Kimura, I. Nakanishi, M. T. Bayliss, K. Iwata, and H. Nagase. 1992. Localization of matrix metalloproteinase 3 (stromelysin) in osteoarthritic cartilage and synovium. Lab. Invest. 66:680-690

21. Dean, D. D., J. Martel-Pelletier, J.-P. Pelletier, D. S. Howell, and J. F. Woessner, Jr., 1989. Evidence for metalloproteinase and metalloproteinase inhibitor imbalance in human osteoarthritic cartilage. J. Clin. Invest. 84:678-685.

22. Hascall, V. C. 1977. Interaction of cartilage proteoglycan with hyaluronic acid. J. Supramolec Str. 7:101-120.

23. Poole, A. R., I. Pidoux, A. Reiner, and L. Rosenberg. 1982. An immunoelectron microscope study of the organization of proteoglycan monomer, link protein, and collagen in the matrix of articular cartilage. J. Cell Biol. 93:921937.

24. Poole A. R., C. Webber, I. Pidoux, H. Choi, and L. C. Rosenberg. 1986 The localization of a dermatan sulfate proteoglycan (DS-PGII) in cartilage and the presence of an immunologically related species in other tissues. J. Histochem. Cytochem.34:619-625.

25. Vogel, K. G., M. Paulsson, and D. Heinegård. 1984. Specific inhibition of type I and type II collagen fibrillogenesis by the small proteoglycan of tendon. Biochem. J. 223:587-597.

26. Scott, J. E. 1988. Proteoglycan-fibrillar collagen interactions. Biochem. J. 252:313-323.

27. Palmoski, M. J., and K. D. Brandt. 1984. Effects of static loading and cyclic compressive loading on articular cartilage plugs in vitro. Arthritis \& Rheum. 27:675-681.

28. van Kampen, G. P. J., J. P. Veldhuijzen, R. Kuijer, R. J. van De Stadt, and C. A. Schipper. 1985. Cartilage response to mechanical force in high-density chondrocyte cultures. Arthritis \& Rheum. 28:419-424.

29. Sah, R. L.-Y., J.-Y. H. Doong, and A. J. Grodzinsky. 1991. Effects of compression on the loss of newly synthesized proteoglycans and proteins from cartilage explants. Arch. Biochem. Biophys. 286:20-29. 